\title{
Notas para un primer paso en la hoja de ruta de la modernización del aparato estatal
}

Some remarks for a first step forward towards the modernization of state apparatus

\section{Diego Pando}

Doctor en Ciencia Política, Universidad Complutense de Madrid.

Director de la Licenciatura en Políticas Públicas y Gobierno de la Universidad Metropolitana para la Educación y el Trabajo y profesor de la Escuela de Administración y Negocios de la Universidad de San Andrés.

Presidente de la Asociación Argentina de Estudios de la Administración Pública. pando.d@umet.edu.ar dpando@udesa.edu.ar
Fecha de recepción:

14.7.16

Fecha de aprobación:

7.9.16

\section{Resumen}

El Plan de Modernización del Estado es la enunciación de un conjunto de lineamientos para una gestión pública más orientada a la satisfacción de las demandas de la ciudadanía. De todos los lineamientos planteados, aquel que menos avances registra durante estos primeros meses de gobierno es el más relevante: la profesionalización del empleo público. Para avanzar en esta dirección un primer paso necesario consiste en focalizar el esfuerzo de la profesionalización en la alta dirección pública.

Palabras clave: modernización - aparatos del estado - gestión pública - recursos humanos - empleo público - Argentina.

\section{Abstract \\ The Plan for the Modernization of the State elicits a number of actions towards a system of public manage- ment more clearly addressed at the satisfaction of citizens' demands. Notwithstanding its relevance to achieve this goal, professionalization of public employment stands as lagging behind the rest of commitments there in. In order to fill this gap it is recommended to put leverage in the professionalization of high-level public management.}

Key-words: modernization - state apparatus - public management - human resources - public employment - Argentina 
El fortalecimiento de la capacidad de gobernar requiere avanzar hacia una gestión pública más orientada a la satisfacción de las crecientes y heterogéneas demandas de la ciudadanía. El Plan de Modernización (PM) presentado en marzo pasado (Decreto 434/2016) es la explicitación de un conjunto de lineamientos que van en esa dirección.

Cualquier estudioso de la administración pública en Argentina coincidiría en la importancia de los ejes planteados en el PM: fortalecer e incorporar infraestructura tecnológica con el fin de facilitar la interacción entre el ciudadano y los diferentes organismos públicos; avanzar en la profesionalización de la función pública; desarrollar un modelo de gestión que haga énfasis en los resultados basado en sistemas de rendición de cuentas que aumenten la transparencia; abrir la evaluación y el control de los programas de las instituciones públicas a la participación de las organizaciones de la sociedad civil; y generar un marco de colaboración con las administraciones públicas provinciales y municipales.

De todas las cuestiones planteadas, aquella que menos avances registra durante estos primeros meses del actual gobierno es sin dudas la más relevante: la profesionalización del empleo público. El desarrollo de un servicio público profesional es una condición esencial en todo intento modernizador dado que, bajo la conducción del nivel político, son los funcionarios los que producen bienes y entregan servicios a la ciudadanía. Sin ellos, ninguna de las demás cuestiones que aparecen en el PM (la tecnología, la orientación por resultados, etc.) podría desarrollarse. Esto además cobra mayor relevancia en un contexto en el cual el Estado contemporáneo es significativamente mayor y más complejo en tamaño y funciones de lo que era en 2003.

Desde una mirada general, en la administración pública nacional se observan burocracias con realidades disímiles y desarticuladas. Así, y además de los núcleos de profesionales con elevada capacidad técnica, se observa a) una burocracia mayoritariamente administrativa (inamovilidad, bajas posibilidades de desarrollo de carrera, salarios poco competitivos con respecto al mercado laboral y, en el mejor de los casos, un ritual de evaluación del desempeño con pocos fines prácticos), b) una estructura temporal de funcionarios superpuesta al aparato administrativo y c) grupos burocráticos como una extensión más del actor político partidario, con cierto poder de veto frente a segmentos profesionales con los cuales muchas veces entra en conflicto.

Esta heterogeneidad se refleja en la existencia de más de 50 regímenes laborales, situación que se torna aún más compleja si a este mosaico de ordenamientos jurídicos se agrega el centenar de regímenes salariales existentes. Si bien esta dispersión normativa ha sido una característica distintiva de las regulaciones del empleo público desde la década del ochenta, los esfuerzos realizados por unificar y profesionalizar la carrera no tuvieron resultados significativos. A modo de ejemplo podemos señalar 
que el Sistema Nacional de Empleo Público creado en 2008 (modificando el Sistema Nacional de la Profesión Administrativa de los noventa) abarca menos del 10\% del empleo público nacional.

Ahora bien: no se puede fortalecer la profesionalización de la burocracia de la noche a la mañana y menos como si fuera un todo homogéneo. Así, y enfatizando que la acción de gobierno no puede consistir en respuestas aleatorias a desafios coyunturales, es necesaria la identificación de prioridades y asignación de recursos en un contexto de altas exigencias por avanzar hacia una gestión comprometida con los resultados. Nada de esto se percibe hasta el momento en la actual gestión de gobierno.

La gradualidad en la formulación e implementación de las reformas, focalizándose en unos problemas primero y en otros después, es clave. Esto se debe no sólo porque los problemas son demasiado complejos y poco estructurados para abordarlos de manera conjunta, sino porque existen limitaciones políticas y técnicas para enfrentarlos.

La gradualidad implica diseñar una hoja de ruta donde las diferentes piezas se despliegan en el mediano y largo plazo, aunque se tenga claridad y acuerdo sólo sobre las iniciales. La pregunta entonces es: ¿por dónde empezar?

Los procesos de reforma del servicio civil en Chile, Perú y Ecuador durante la última década, con sus matices y desafios pendientes, muestran un camino que vale la pena tener en cuenta: focalizar el esfuerzo de la profesionalización en la alta dirección pública. Sin pretender copiar acríticamente desconociendo el contexto político-institucional en el cual se enmarcan, el Sistema de Alta Dirección Pública vigente en Chile desde 2003, el Cuerpo de Gerentes Públicos existente desde 2008 en Perú y el Programa de Ejecutivos de Excelencia creado en 2013 en Ecuador, constituyen tres experiencias para ser consideradas por sus impulsos reformadores.

Por ser un espacio particularmente sensible en donde confluyen el mundo de la política y el de la expertise técnica, la profesionalización del segmento superior de la burocracia debe ser abordada como una parte diferenciada (Cortázar Velarde, Lafuente, Longo y Schuster, 2014). Necesita un marco normativo propio y un modelo meritocrático pero más flexible que el que corresponde al resto de los niveles para avanzar en relación a 1) la definición de cargos y perfiles en base a modelos por competencias (conocimientos técnicos, habilidades y actitudes); 2) la gestión del ingreso por concursos, movilidad y desvinculación de los directivos; 3) el diseño y evaluación del rendimiento; 4) la retribución competitiva con el sector privado; 5) la formación y la definición de itinerarios de carrera. Tan importante como avanzar en cada uno de estos elementos, es hacerlo de manera coordinada. 
El primer motivo que justifica la focalización es la importancia que tiene el segmento directivo para el buen desempeño institucional. Se trata de un segmento que atraviesa a todo el sector público y que no puede estar sujeto a la incertidumbre y discrecionalidad con baja responsabilidad propias de la dinámica patrimonialista más característica del siglo XIX que del siglo XXI. La alta dirección es un estamento transversal que se despliega por todo el sector público en el que existe conocimiento especializado, inversión en aprendizaje y memoria institucional. Como señalan Cortázar Velarde, Fuenzalida y Lafuente (2016) a partir del análisis de las experiencias de Chile y Perú, la llegada de los directivos públicos seleccionados trajo mejoras en el establecimiento de mecanismos de coordinación efectiva entre áreas, la formalización de procedimientos de trabajo, la renovación de estructuras organizativas y un uso más eficiente de los recursos disponibles.

El segundo motivo tiene que ver con la viabilidad política del proceso. Ninguna iniciativa de cambio suele ser prolija y esta no es la excepción dado que implica la renuncia de los políticos a su capacidad de seleccionar discrecionalmente a los directivos públicos. Sin embargo, focalizar en la alta dirección pública disminuye los riesgos de una confrontación con poderosos actores con capacidad de veto en este juego como los sindicatos de trabajadores estatales.

Finalmente (pero no menos importante en función de la viabilidad económica), focalizar en la alta dirección pública permite una estimación razonable de los costos financieros de la reforma, los cuales debieran ser relativamente menores en comparación al costo total de la planta de empleados públicos. De esta forma, y dado que la participación de las instituciones responsables del presupuesto es fundamental, disminuye la posibilidad de que se activen vetos fiscales que suelen estar entre las principales trabas de cualquier proceso de reforma.

Un requisito previo fundamental para avanzar en esta agenda de reforma y sostener el esfuerzo inter-temporalmente, es el fortalecimiento de la Secretaría de Empleo Público, dependiente del Ministerio de Modernización, como organismo rector del servicio civil (una especie de director de orquesta). Orientado a la definición de una secuencia o plan temporal de acciones viables política y técnicamente, con objetivos factibles e indicadores pertinentes, articulando con las áreas de recursos humanos de los organismos públicos, la responsabilidad central del organismo rector es constituirse en el nódulo de un sistema de coordinación que asegure coherencia y complementariedad de las acciones orientadas a la definición de cargos y perfiles, a la gestión del empleo de los directivos, al diseño y evaluación del rendimiento, a la retribución y a la formación y la definición de itinerarios de carrera.

Una última consideración a tener en cuenta para el proceso de reforma. Es fundamental priorizar la mejora efectiva del segmento directivo y no solo el perfeccionamiento 
del marco normativo. Existe abundante evidencia empírica reciente acerca de que es más sencillo introducir cambios a través de normativa que asegurar su implementación (Cortázar Velarde, Lafuente y Schuster, 2014). Si bien la puesta en práctica de un proceso de estas características no tiene las luces de la formulación, se trata de la etapa más importante dado que es ahí donde las cosas realmente suceden (o debieran suceder).

En síntesis: bajo un enfoque situacional adaptable a contingencias, el PM debe (re) definir prioridades de acción con énfasis en la profesionalización del empleo público dada la relevancia que tiene para fortalecer la calidad de bienes y servicios que brinda el aparato estatal. En este sentido, y dado que resulta imposible imaginar un servicio civil profesional si su parte superior y más influyente en su funcionamiento y sus resultados se mantiene sometida a la excesiva discrecionalidad política, focalizar el esfuerzo en la alta dirección pública es una buena forma de comenzar.

Los caminos de la modernización del Estado en general y el de la profesionalización del empleo público (con especial acento en la alta dirección pública) en particular, no están libres de obstáculos, en el sentido que implican afrontar tensiones, negociaciones, desafios e incertidumbres. Pero sin dudas son caminos que valen la pena ser transitados. El reconocimiento de esta conflictividad inherente a este proceso sirve para llamar la atención de los riesgos asociados a promover esfuerzos guiados pura y exclusivamente por la apelación a la buena voluntad. 


\section{Referencias bibliográficas}

Cortázar Velarde, Juan Carlos, Mariano Lafuente y Christian Schuster, (2014): “Estrategias para avanzar en la modernización del servicio civil en América Latina”, en Juan Carlos CortázarVelarde, Mariano Lafuente y Mario Sanginés (editores): Al servicio del ciudadano: una década de reformas del servicio civil en América Latina (2004-13), Washington DC: Banco Interamericano de Desarrollo.

Cortázar Velarde, Juan Carlos, Mariano Lafuente, Francisco Longo y Christian Schuster, (2014): "La agenda a futuro: estrategias y tareas clave para mejorar el servicio civil en América Latina”, en Juan Carlos Cortázar Velarde, Mariano Lafuente y Mario Sanginés (editores): Al servicio del ciudadano: una década de reformas del servicio civil en América Latina (2004-13). Washington DC: Banco Interamericano de Desarrollo.

Cortázar, Juan Carlos, Javier Fuenzalida y Mariano Lafuente (2016): Sistemas de mérito para la selección de directivos públicos ¿Mejor desempeño del Estado? Un estudio exploratorio, nota técnica. Washington DC.: Banco Interamericano de Desarrollo, IDB-TN-1054.

\section{Cómo citar este artículo}

Pando, Diego: Notas para un primer paso en la hoja de ruta de la modernización del aparato estatal. Revista Perspectivas de Políticas Públicas (2016) Vol. 6, No 11:17-22. 